\title{
Hypokalaemia and hyperkalaemia
}

\author{
A Rastergar, M Soleimani
}

\begin{abstract}
Disturbances in potassium homoeostasis presenting as low or high serum potassium are common, especially among hospitalised patients. Given the fact that untreated hypokalaemia or hyperkalaemia is associated with high morbidity and mortality, it is critical to recognise and treat these disorders promptly. In this article, normal potassium homoeostasis is reviewed initially and then a pathophysiological approach to work-up and management of hypokalaemia and hyperkalaemia is presented. Recent advances with respect to the role of kidney in handling of the potassium, the regulation of renal ion transporters in hypokalaemia, and treatment of hypokalaemia and hyperkalaemia will be discussed.

(Postgrad Med f 2001;77:759-764)
\end{abstract}

Keywords: hypokalaemia; hyperkalaemia; potassium

Potassium homoeostasis

Potassium is the most abundant cation in the body. It is predominantly restricted to the intracellular space, such that only $2 \%$ is located extracellularly and the remaining $98 \%$ is in the intracellular compartment. The ratio of intracellular to extracellular potassium $(\mathrm{Ki} / \mathrm{Ke})$ is the major determinant of resting membrane potential, and is regulated primarily by the sodium-potassium ATPase pump located on the plasma membrane of most cells. Although extracellular potassium accounts for only $2 \%$ of total body potassium, it has a major effect on the ratio of $\mathrm{Ki} / \mathrm{Ke}$ and through that on the resting membrane potential. As a result, serum potassium is normally regulated around the narrow range of 3.5-5.0 mmol/1.

The daily intake of potassium in the western diet is between $80-120$ mmol. The kidney is the major route of potassium excretion, accounting for $90 \%$ of potassium loss daily. The remaining $10 \%$ is excreted through the gastrointestinal tract. The kidney is, therefore, responsible for long term potassium homoeostasis, as well as the serum potassium concentration. On short term basis, serum potassium is also regulated by the shift of potassium between the intracellular and extracellular

2000

Accepted 14 June 2001

ssium distribution between intracellular and extracellular Table 1 Regut
compartment

\begin{tabular}{lll}
\hline Regulators & Mechanism of action & Potassium shift into cells \\
\hline Insulin & Activation of sodium-potassium & Increase \\
& ATPase & \\
Catecholamines & Activation of $\beta_{2}$ receptors & Increase \\
& Activation of $\alpha$ receptors & Decrease \\
Mineralocorticoids & Unknown & Mild increase \\
Parathormone & Unknown & Mild decrease \\
Acid-base changes & Exchange of H+ for K+ & See table 2 \\
Hyperosmolality & Solvent drags & Shifts potassium extracellularly \\
\hline
\end{tabular}

compartments. Humans, as carnivorous animals, consume large amount of potassium intermittently. Dietary potassium, which is rapidly absorbed by the gut, could increase serum potassium dramatically. However, several physiological mechanisms quickly shift the potassium intracellularly, allowing slow excretion of potassium by the kidney, and maintenance of normal potassium homoeostasis. ${ }^{1}$

Normal physiological regulators, insulin and catecholamines, are stimulated by ingestion of food containing glucose and potassium. These hormones are essential in shift of potassium intracellularly, depositing it primarily in the liver and striated muscle cells. ${ }^{2}$ Catecholamines, by acting through different receptors, have different effect on potassium deposition. $\beta_{2}$-stimulation results in a shift of potassium into the cell, while $\alpha$-stimulation has the opposite effect. ${ }^{3}$ The effect of mineralocorticoids and parathyroid hormone in internal potassium homoeostasis is minimal at best. In addition to these physiological regulators, internal potassium homoeostasis is also affected by changes in acid-base and osmolarity. Sudden changes in osmolarity, by shifting the water out of cell, creates a solvent drag phenomenon, and helps push potassium out of the cell, resulting in a rise in serum potassium (table 1). The effect of acid-base status is much more complicated and depends on the nature of the disorder (box 1). Although, the rule of thumb has been that for each 0.1 unit change in $\mathrm{pH}$, there is a $0.6 \mathrm{mmol} / 1$ change in serum potassium, this is a very crude approximation and varies greatly by the nature of acid-base disorders. For example organic acidosis as seen in diabetic ketoacidosis or lactic acidosis result in little or no change in serum potassium while non-organic (mineral) acidosis, such as acidosis of renal failure, has the greatest effect. Other acid-base disorders shift potassium minimally. ${ }^{45}$

\section{Box 1: Effect of acid-base disorders on serum potassium}

- For any $\mathrm{pH}$ change the effect of acidaemia is greater than alkalaemia.

- Non-organic (mineral acidosis) results in a shift of $0.24-1.7 \mathrm{mmol} / 1$ per 0.1 unit $\mathrm{pH}$ change.

- Organic acidosis has little to no effect on potassium shift.

- Respiratory and metabolic alkalosis and respiratory acidosis result in similar small shift of potassium into and out of cell respectively (0.1-0.4 $\mathrm{mmol} / 1$ on average).

- In chronic acid-base disorders the final potassium reflects primarily the effect on renal handling of potassium and to lesser extent of transcellular shift. 
Renal handling of potassium

The filtered potassium (around 700-800 $\mathrm{mmol} /$ day) is largely reabsorbed by proximal nephron segments, including proximal convoluted tubules and thick limb of Henle. The potassium that is excreted is, therefore, a result of secretion by distal segments, predominantly distal convoluted tubule and the collecting duct. Transport studies in these latter tubule segments have demonstrated that potassium secretion is accomplished via apical potassium channels. The secretion of potassium in these nephron segments is indirectly but tightly coupled to sodium reabsorption via the amiloridesensitive sodium channel; increased sodium reabsorption increases whereas decreased sodium reabsorption decreases potassium secretion. It is this secretory ability of the potassium channels in the distal segments which regulates the excretion of potassium. As a result, any condition that decreases the activity of renal potassium channels results in hyperkalaemia (for example, amiloride intake or aldosterone deficiency) whereas their increased activity results in hypokalaemia (for example, primary aldosteronism or Liddle's syndrome).

In summary, kidney is the major regulator of long term potassium homoeostasis and serum potassium. However, on short term basis, insulin and catecholamines, among others, regulate serum potassium through changes in transcellular distribution of potassium.

\section{Hyperkalaemia}

Hyperkalaemia is defined as serum potassium greater than $5.0 \mathrm{mmol} / \mathrm{l}$. True hyperkalaemia should however be distinguished from pseudohyperkalaemia, a rise in serum potassium secondary to release of intracellular potassium during phlebotomy or storage of blood sample. During phlebotomy the combination of venous occlusions and hand clinching could result in potassium release locally. If this is suspected, a blood sample should be drawn from a free flowing vein without fist clinching. Potassium can also be released in test tube by haemolysis, severe thrombocytosis (usually $>900 \times 10^{10} / 1$ platelets) or leucocytosis (leucocytes $>70 \times 10^{9} /$ 1). If this is suspected the measurement should be repeated using fresh heparinised blood drawn carefully to prevent haemolysis.

The incidence of hyperkalaemia in hospitalised patients varies depending on the level of potassium used from $1.4 \%$ to $10 \%$. In the largest study in a single hospital overall incidence of hyperkalaemia (defined as serum potassium $>6.0 \mathrm{mmol} / \mathrm{l}$ ) was $1.4 \%$. Potassium supplementation and potassium-sparing diuretics account for about one third of the cases. Severe hyperkalaemia is more common in older patients with underlying renal insufficiency treated with potassium supplementation. Hyperkalaemia accounts for approximately 1:1000 deaths in hospitalised patients (reviewed by Ponce et $a l^{6}$ ).

CLASSIFICATION OF HYPERKALAEMIA

Hyperkalaemia could be due to transcellular shift, increase in intake, and/or decrease in output (box 2). Transcellular shift is often due to

\section{Box 2: Classification of hyperkalaemia}

(1) Spurious hyperkalaemia

- Due to high platelet and/or leucocyte count.

- Due to muscular activity during venepuncture.

(2) Transcellular shift of potassium

- Acidaemia (for example, acute renal failure).

- Hyperosmolality (for example, severe hyperglycaemia).

- $\beta_{2}$-blockers (for example, propranolol).

- Insulin deficiency (for example, type I diabetes mellitus).

(3) Increase intake

- Infusion of potassium containing solutions.

- Increase potassium intake in patients with defect in potassium excretion.

(4) Decrease renal excretion

- Mineralocorticoid deficiency: (a) Addison's disease, (b) isolated aldosterone deficiency, (c) renin deficiency (for example, diabetic nephropathy), (d) angiotensin II receptor blockers, (e) angiotensin converting enzyme inhibitors, (f) use of non-steroidal anti-inflammatory drugs.

- Resistance to mineralocorticoids effect: (a) tubulointerstitial disease, (b) high dose mineralocorticoids antagonists (for example, spironolactone, trimethoprim).

- Severe renal failure.

metabolic acidosis; however, a sudden rise in osmolality, especially in association with insulin deficiency, could result in significant hyperkalaemia. $\beta$-blockers alone are rarely associated with significant hyperkalaemia, however, they could play a contributory part.

Given the renal ability to excrete large amount of potassium, increase in intake could result in hyperkalaemia, only if associated with subtle or overt defect in potassium excretion. Salt substitutes, which may contains as much as $200 \mathrm{mmol}$ of potassium per tablespoon, are major hidden sources of ingested potassium. Hyperkalaemia can also occur by infusion of potassium containing solution at a rate that can not be handled by transcellular shift and/or renal excretion (see below under treatment of hypokalaemia). The most important cause of hyperkalaemia is, however, decrease in renal potassium excretion. This is seen in many disorders including mineralocorticoid deficiency, such as Addison's disease or resistance to the effect of aldosterone such as seen in patients on aldosterone antagonist drugs (box 2). Trimethoprim, a commonly used antimicrobial drug, is an important cause of hyperkalaemia in patients with mild renal failure. ${ }^{7}$ Although this side effect is more common on high dose intravenous therapy, it does occur on regular oral dose. Patients with renal failure can often maintain near normal serum potassium unless glomerular filtration rate decreases below 15 
$\mathrm{ml} / \mathrm{min}$. However, a significant number of patients with renal disease have low aldosterone levels with or without low renin level, or have resistance to aldosterone effect. This group presents with mild to moderate renal failure and hyperkalaemia, often in association with renal tubular acidosis (type IV). ${ }^{8}$

CLINICAL PRESENTATION

Hyperkalaemia is often asymptomatic and is discovered on routine laboratory tests. Patients with severe hyperkalaemia (potassium $>6.5$ $\mathrm{mmol} / \mathrm{l}$ ) may, however, present with generalised weakness, paralysis, and cardiac arrhythmia, including cardiac stand still and sudden death. In general, the severity of clinical presentation does correlate with the severity of hyperkalaemia. Changes in the electrocardiogram (ECG) also reflect the severity of hyperkalaemia. In mild to moderate hyperkalaemia, changes in the ECG are subtle and often limited to peaking of the T-wave. If hyperkalaemia is more severe, prolongation of $\mathrm{PR}$ and QRS interval followed by loss of $\mathrm{P}$ wave and marked widening of QRS is seen. In extreme hyperkalaemia, the ECG shows sine wave, often followed by ventricular fibrillation. Although some patients show a gradual progression of ECG findings, many progress rapidly without warning. Therefore, hyperkalaemia in association with ECG changes is a true medical emergency.

WORK-UP OF HYPERKALAEMIA

It is important to stress that in severe hyperkalaemia diagnostic work-up should be postponed until hyperkalaemia is treated. In other patients, if the cause of hyperkalaemia is not evident from the initial work-up, a stepwise approach is recommended. The first step should be to evaluate the adequacy of the renal response to hyperkalaemia. Potassium excretion is primarily through potassium secretion in the cortical collecting duct. Urinary potassium concentration is however greatly affected by the amount of water reabsorbed in the collecting duct. To evaluate adequacy of renal response it is therefore important that urinary potassium to serum potassium ratio be corrected for urinary concentration. This is simply done by dividing the ratio of urinary potassium to serum potassium by the ratio of urinary osmolality to serum osmolality (urinary potassium:serum potassium/urinary osmolality:serum osmolality). This ratio, referred to as transtubular potassium gradient or TTKG, is $>5$ and often 7 in hyperkalaemia and $<1$ in hypokalaemia. ${ }^{910}$ It is worth mentioning that the cut off values of $>5$ or $<1$ are indicative of a non-renal cause for high or low potassium, respectively. This formula, however, can not be used if urine is more dilute than the serum or contains very little sodium. ${ }^{9}{ }^{10}$

The next step is to establish if low urinary potassium excretion is due to low aldosterone or to resistance to aldosterone effect by measuring serum aldosterone level. In patients with underlying renal disease hyperkalaemic renal tubular acidosis is a very common finding and is often due to hypoaldosteronism with or without a low renin level. In these patients we commonly do not embark on a costly and detailed evaluation and focus on long term treatment. However in patients with normal renal function, and especially in patients suspected of primary adrenal failure, a complete work-up including measurement of aldosterone and cortisol level is mandatory.

One of the more overlooked and less well understood causes of hyperkalaemia is the use of prostaglandin inhibitors or non-steroidal anti-inflamatory drugs (NSAIDS). Studies have shown that the use of NSAIDS, specifically in conditions associated with raised basal renal prostaglandins such as liver cirrhosis or mild renal insufficiency, can cause hyperkalaemia by causing hypoaldosteronism. Two independent mechanisms are responsible for this hyperkalaemia; first is the direct inhibition of renin synthesis by prostaglandin inhibition. ${ }^{11}$ The second mechanism is indirect and is via enhanced reabsorption of sodium and chloride in the thick ascending limb which can result in volume expansion and as a result suppress renin and aldosterone. ${ }^{11}$

If renal response to hyperkalaemia is appropriate (TTKG >5), increase in potassium intake, or transcellular shift of potassium should be suspected. As indicated above, transcellular shift most commonly occurs in the setting of metabolic acidosis, hyperosmolality, and/or insulin deficiency. If transcellular shift is ruled out, hyperkalaemia is probably due to an increase in potassium intake, which should be established by a careful dietary (including food supplements) and drug history.

MANAGEMENT OF HYPERKALAEMIA

The initial management should be dictated by the changes in ECG. In the presence of ECG changes, hyperkalaemia should be considered an emergency and treatment should begin immediately with calcium gluconate infusion. This should be followed by use of insulin and glucose or albuterol to help shift potassium into the cell before a more definitive treatment, cation exchange resin (sodium or calcium polysteryene sulphone resin) and/or dialysis, is used to remove potassium from the body. Insulin and albuterol have an additive effect in lowering serum potassium. ${ }^{12}$ Table 2 summarises emergency treatment for severe hyperkalaemia. Although sodium bicarbonate use has fallen out of favour in patients on dialysis, it should be considered in patients with significant acidaemia where it is expected that infusion of bicarbonate would increase serum $\mathrm{pH}$ significantly. ${ }^{13}$ Cation exchange resin mixed with sorbitol should be used orally if hyperkalaemia is not life threatening, however resin mixed with water (and not sorbitol) can be repeated hourly for rapid removal of potassium. It should be remembered that each gram of sodium polystyrene resin (Kayexalate) removes $0.5-1.0 \mathrm{mmol}$ of potassium in exchange for $2-3 \mathrm{mmol}$ of sodium. Therefore Kayexalate use is associated with significant sodium infusion and can result in volume overload. In addition several cases of colonic perforation have been reported in 
Table 2 Treatment of hyperkalaemia

\begin{tabular}{llll}
\hline Mechanism of action & Therapy & Onset/duration & Dose \\
\hline I. Membrane stabilisation & Calcium & $1-3 \mathrm{~min} / 30-60 \mathrm{~min}$ & Calcium gluconate $10 \% 10 \mathrm{ml}$ iv \\
II. Shift of potassium intracellularly & Insulin & $20 \mathrm{~min} / 4-6$ hours & $10 \mathrm{U}$ regular insulin iv with $50 \mathrm{ml} 50 \%$ dextrose \\
& $\beta_{2}$-adrenergic agonist & $20 \mathrm{~min} / 2-4$ hours & $\begin{array}{l}\text { Dose depending on the type of agonist used } \\
15 \mathrm{~g} \text { every six hours orally or 30-60 g by } \\
\text { III. Removal of potassium }\end{array}$ \\
& Sodium or calcium polystyrene sulphone & $1-2 \mathrm{hours} / 4-6$ hours & $\begin{array}{l}\text { retention enema } \\
2-3 \text { hours haemodialysis }\end{array}$ \\
\hline
\end{tabular}

iv $=$ intravenous.

patients treated with Kayexalate mixed with sorbitol. ${ }^{14}$

After the acute treatment of hyperkalaemia, a long term plan should be devised to prevent recurrence of hyperkalaemia. Initially the treatment should be directed toward correction of the underlying cause of hyperkalaemia (such as replacement therapy in patients with Addison's disease). If hyperkalaemia is due to use of drug (such as aldosterone antagonists or potassium supplements), these should be discontinued. If hyperkalaemia is due to tubular defect in potassium secretion in association with renal failure, several therapeutic manoeuvres should be considered including:

- Hydration and volume expansion to increase urine flow rate and sodium delivery to exchange site.

- Use of loop diuretics to increase sodium delivery and stimulate potassium excretion.

- Restriction of dietary potassium intake to approximately $60 \mathrm{mmol} /$ day.

- Use of oral mineralocorticoids such as fludrocortisone in supraphysiological doses. Most patients can be managed without use of fludrocortisone, however in some patients use of this drug in doses of $0.4-1.0 \mathrm{mg}$ maybe needed. Hypertension and/or oedema formation maybe a limiting side effects in use of this drug.

\section{Hypokalaemia}

Hypokalaemia is probably the most common electrolyte abnormality in hospitalised patients. It is usually defined as a serum potassium of less than $3.5 \mathrm{mmol} / \mathrm{l}$. Patients with mild hypokalaemia (serum potassium 3.0-3.5 $\mathrm{mmol} / \mathrm{l}$ ) usually have no symptoms. However, with more severe hypokalaemia (serum potassium of less than $2.5 \mathrm{mmol} / \mathrm{l}$ ), generalised weakness can occur. In addition, patients with severe hypokalaemia can develop muscle necrosis (rhabdomyolysis) and paralysis. Both mild and severe hypokalaemia can increase the incidence of cardiac arrhythmias.

CLASSIFICATION OF HYPOKALAEMIA

Hypokalaemia can result from increased loss, transcellular shift, or decreased intake of potassium. ${ }^{348}$ Increased potassium loss (through the kidney or gastrointestinal tract) is the most common cause of hypokalaemia. Less frequently, hypokalaemia can occur as a result of shift of potassium from the extracellular space into cells. Rarely, hypokalaemia can result from decreased intake of potassium.

Increased potassium loss, which is the most common cause of hypokalaemia, occurs mostly in patients who are on diuretics (thiazide or loop diuretics) or in patients with gastrointestinal diseases (diarrhoea). Thiazide and loop diuretics increase delivery of sodium to the collecting ducts, where it is reabsorbed via the amiloride-sensitive sodium channel, therefore creating a favourable gradient for potassium secretion via potassium channels. In addition, volume depletion that results from these diuretics increases aldosterone (by activation of renin-angiotensin-aldosterone pathway), further increasing potassium secretion via the secretory potassium channels in the collecting ducts. Increased aldosterone, in addition, can cause metabolic alkalosis by increasing hydrogen mediated bicarbonate reabsorption in the collecting duct. This latter phenomenon can worsen the diuretic-induced hypokalaemia by increasing potssium shift into cells. The diuretic acetazolamide, which causes metabolic acidosis by decreasing bicarbonate reabsorption in the proximal tubule, increases potassium excretion by increasing the delivery of sodium and bicarbonate to the distal nephrons. Hypokalaemia can also be due by increased loss in the stool in patients taking large doses of laxatives or having diarrhoea.

Hypokalaemia due to potassium shift into cells is caused by medications, hormonal dysregulation, or raised blood $\mathrm{pH} .{ }^{34815}$ These medications include $\beta_{2}$-sympathomimetics (that is brochodilators such as albuterol) or phosphodiesterase inhibitors (that is theophylline and caffeine), exogenous insulin and rarely calcium channel blockers. Decreased potassium intake (less than $1 \mathrm{~g} /$ day), while rare, can lead to hypokalaemia. This is due to obligatory potassium loss of $10-15 \mathrm{mmol} /$ day by the kidney despite a low potassium intake.

Among other disorders causing hypokalaemia magnesium depletion needs special emphasis. ${ }^{14}$ Magnesium depletion, which is caused by either decreased dietary intake or increased loss, is a common electrolyte disorder in hospitalised patients. It can cause severe hypokalaemia by increasing renal potassium loss. The exact mechanism is, however, remains unclear. Hypokalaemia is also a common finding in patients with raised serum aldosterone either secondary to the activation of the renin-angiotensin system (Bartter's syndrome or Gitelman's syndrome) or due to overproduction by aldosterone-producing tumours (primary aldosteronism).

CLINICAL PRESENTATION OF HYPOKALAEMIA

Similar to hyperkalaemia, hypokalaemia is often asymptomatic. This is specifically true in patients with mild hypokalaemia (serum potassium 3.0-3.5 mmol/l). Patients with more severe hypokalaemia (serum potassium of less 
than $2.5 \mathrm{mmol} / \mathrm{l}$ ) usually present with generalised weakness and, in some cases, ascending paralysis. In addition, severe hypokalaemia can precipitate rhabdomyolysis which manifests as muscle tenderness and swelling. Cardiac arrhythmias are common in hypokalaemia, specifically in patients with underlying heart disease or on digoxin. In moderate to severe hypokalaemia changes in ECG are minimal and is often limited to the presence of a $U$ wave.

RENAL SYNDROMES ASSOCIATED WITH

HYPOKALAEMIA

In addition to the above clinical symptoms, hypokalaemia can cause several distinct renal syndromes as will be discussed below.

\section{Nephrogenic diabetes insipidus}

Hypokalaemia can impair urinary concentrating mechanism and result in nephrogenic diabetes insipidus. Patients with nephrogenic diabetes insipidus due to hypokalaemia present with polyuria and polydipsia. Molecular studies have demonstrated that potassium depletion causes downregulation of the water channel aquaporin 2 in the collecting duct, therefore impairing the renal concentrating mechanism and resulting in polyuria. ${ }^{16}$

\section{Metabolic alkalosis}

Hypokalaemia can contribute to the maintenance of metabolic alkalosis in several disease states (such as vomiting) by enhancing bicarbonate absorbing ability of renal tubules. ${ }^{17-20}$ This in turn decreases the ability of the kidney to excrete the excess bicarbonate and as a result maintains the plasma bicarbonate at a raised level. ${ }^{17}$ Functional and molecular studies in luminal and basolateral membranes of kidney proximal tubules and in microperfused kidney nephrons have demonstrated that hypokalaemia upregulates the expression of bicarbonate absorbing transporters in proximal tubules and cortical and medullary collecting ducts. ${ }^{17-20}$ There is also evidence in support of hypokalaemia being involved in the generation of metabolic alkalosis in human by increasing ammoniagenesis. ${ }^{17}$

\section{Enhanced renal chloride excretion}

Hypokalaemia increases urinary chloride excretion. ${ }^{19}{ }^{20}$ Functional and molecular studies in the kidney have demonstrated that renal chloride wasting in hypokalaemia is due to suppression of the apical sodium-potassiumchloride cotransporter in the thick limb of Henle and the apical sodium-chloride cotransporter in the distal convoluted tubule. ${ }^{21}$ It is possible that by increasing renal chloride excretion, hypokalaemia can result in hypochloraemia, which in turn can contribute to the maintenance of metabolic alkalosis in pathophysiological states. ${ }^{17}$

WORK-UP OF HYPOKALAEMIA

In working up patients with hypokalaemia a combination of common sense as well as pathophysiology should be used (box 3). Transient short term hypokalaemia is usually caused by a potassium shift due to medication or alkalaemia, however prolonged hypokalaemia is commonly due to renal or gastrointestinal loss of potassium. The most common causes of hypokalaemia in clinical practice are due to diuretics and gastrointestinal loss secondary to diarrhoea and/or vomiting. These aetiologies should therefore be considered first before exhaustive and sophisticated work-up is initiated. In other patients the initial step is to see if hypokalaemia is in association with systemic hypertension or not. In the former group hypokalaemia is associated with a high mineralocorticoid effect due to high aldosterone (as in primary aldosteronoma or renal artery stenosis) or cortisol as in Cushing's disease or hyperactive sodium channel as in Liddle's syndrome. Measurement of the renin, aldosterone, and cortisol concentrations under appropriate conditions would help in differentiating among these aetiologies. In normotensive patients, hypokalaemia could be secondary to overt or occult gastrointestinal loss or due to renal potassium wasting. Although low urinary potassium (less than $15 \mathrm{mmol} / \mathrm{l}$ ) would favour gastrointestinal loss, high urinary potassium is seen in patients with vomiting or diarrhoea due to secondary elevation in the aldosterone level and is therefore not very helpful. In normotensive patients with renal potassium wasting, a low serum bicarbonate would favour the diagnosis of renal tubular acidosis, while a high serum bicarbonate is compatible with the high mineralocorticoid effect seen in patients with Bartter's or Gitelman's syndromes. Magnesium deficiency can result in renal potassium wasting and is often seen in alcoholics who are also nutritionally depleted. Diuretic and/or laxative abuse often mimics these rare syndromes and should be considered in any adult patient with hypokalaemia of unknown aetiology and ruled out by urinary test for specific diuretics and stool test for phenolphthalein.

MANAGEMENT OF HYPOKALAEMIA

The management of hypokalaemia is almost always by potassium replacement, with the amount of potassium supplement depending on the severity of hypokalaemia (box 4). The potassium can be given orally (in mild to moerate hypokalaemia) or intravenously (in severe hypokalaemia). When given intravenously, the rate of potassium administration should not exceed $20 \mathrm{mmol} / \mathrm{hour}$. To calculate the amount of potassium supplement, one should have an estimate of the potassium deficit. On average, a reduction of serum potassium by $0.3 \mathrm{mmol} / 1$ suggests a total body deficit of $100 \mathrm{mmol}$. Based on this formula, a patient with a serum potassium of $2.6 \mathrm{mmol} / \mathrm{l}$ needs at least $300 \mathrm{mmol}$ of potassium for the correction of the deficit. In calculating the total body potassium deficit one has to consider factors that can independently affect serum potassium. A patient with a serum potassium of $2.6 \mathrm{mmol} / 1$ has less total body deficit at blood $\mathrm{pH}$ of 7.5 than 7.3. The reason is that alkaline serum $\mathrm{pH}$ (that is, 7.5) can independently lower the serum potassium by intracellular shift. 


\section{Box 3: Work-up of hypokalaemia}

(1) Acute hypokalaemia (less than 12 hours of onset)

- Alkalosis (metabolic or respiratory).

- Insulin therapy (for example, in severe hyperglycaemia).

- $\beta_{2}$-stimulant (for example, albuterol).

(2) Chronic hypokalaemia (more than 24 hours of onset)

- With normal blood pressure.

(A) Increased potassium loss through gastrointestinal tract: (i) diarrhoea, (ii) laxatives.

(B) Increased potassium loss through kidney: (i) diuretics, (ii) hypomagnesaemia, (iii) renal tubular acidosis (proximal and distal), (iv) genetic defects (for example, Bartter's syndrome, Gitelman's syndrome).

- With high blood pressure.

(A) Increased aldosterone: (i) primary aldosteronism (low renin), (ii) renal artery stenosis (high renin), (iii) Cushing's disease (high renin).

(B) Normal or low aldosterone: (i) hyperactive sodium channel (Liddle's syndrome), (ii) increased liquorice intake.

\section{Box 4: Treatment of hypokalaemia}

(1) Intravenous potassium (as potassium chloride)

- Usually reserved for severe hypokalaemia (serum potassium of $<2.6 \mathrm{mmol} / \mathrm{l}$ ).

- The rate of should not exceed 20 $\mathrm{mmol} /$ hour.

(2) Oral potassium

- Potassium chloride: $40-100 \mathrm{mmol} /$ day in divided doses.

- Potassium phosphate (in patients with hypokalaemia and hypophsphataemia).

- Potassium bicarbonate (in patients with acidosis).

\section{Box 5: Selected bibliography}

- Rastegar A, DeFronzo RA. Disorders of potassium and acid-base metabolism in association with renal disease. In: Schrier RW, Gottschalk CW, eds. Diseases of the kidney. 6th Ed. Boston: Little, Brown, 1997: 2452-77.

- Halperin M, Kamel KS. Potassium. Lancet 1998;352:135-40.

- Gennari FJ. Hypokalemia. N Engl f Med 1998;339:451-7.

In addition to potassium chloride, which is commonly used in treating hypokalaemias, potassium phosphate and potassium bicarbonate can be used in certain conditions. Potassium phosphate can be used in patients with combined potassium and phosphate depletion (for example in patients with liver cirrhosis or diabetic ketoacidosis). Potassium bicarbonate can be used in patients with potassium depletion and metabolic acidosis (for example in distal renal tubular acidosis). Aside from intravenous potassium chloride for severe hypokalaemia, mild or moderate hypokalaemia (see above) can be treated with oral potassium chloride. Usually, 50 to $100 \mathrm{mmol}$ of potassium chloride is required per day to maintain serum potassium concentration within the normal range in patients with increased potassium loss (that is, in patients receiving a diuretic).

1 Rosa RM, Williams ME, Epstein FH. Extrarenal potassium metabolism. In: Seldin DW, Giebisch G, eds. The kidney, physiology and pathophysiology. New York: Raven, 1992: 2165-90.

2 DeFronzo RA, Felig P, Ferrannini E, et al. Effect of graded doses of insulin on splanchnic and peripheral potassium metabolism in man. Am f Physiol 1980;238:E421-7.

3 Brown MJ, Brown DC, Murphy MB. Hypokalemia from beta- 2 receptor stimulation by circulating epinephrine. $N$ Engl f Med 1983;309:1414-19.

4 Androgue HJ, Madias NE. Changes in plasma potassium concentration during acute acid-base disturbances. Am f Med 1981;71:456-67.

5 Fulop M. Serum potassium in lactic and keto acidosis. $N$ Engl f Med 1979; 300:1087-90.

6 Ponce SP, Jennings AE, Madias NE, et al. Drug-induced hyperkalemia. Medicine 1985;64:357-70.

7 Velazquez H, Perazella MA, Wright FS, et al. Renal mechanism of trimethoprim-induced hyperkalemia. Ann Intern Med 1993;119:293-301.

8 Dubose TD Jr. Hyperkalemic hyperchloremic metabolic acidosis: pathophysiologic insight. Kidney Int 1997;51:591602.

9 Ethier JH, Kamel KS, Magner PO, et al. The transtubular potassium concentration in patients with hypokalemia and hyperkalemia. Am F Kidney Dis 1990;15:309-15.

10 Kamel KS, Quaggin S, Scheich A, et al. Disorders of potassium homeostasis: an approach based on pathophysiology. Am 7 Kidney Dis 1994;24:597-613.

11 Wright FS, Giebisch G. Regulation of potassium excretion. In: Seldin DW, Giebisch G, eds. The kidney: physiology and pathophysiology. 2nd Ed. New York: Raven Press, 1992: 2209-48.

12 Allon M, Copkney C. Albuterol and insulin for treatment of hyperkalemia in hemodialysis patients. Kidney Int 1990;38: 869-72.

13 Blumberg A, Weidman P, Shaw S, et al. Effect of various therapeutic approaches on plasma potassium and major therapeutic approaches on plasma potassium and major
regulating factors in terminal renal failure. Am $\mathcal{F}$ Med 1988; 85:507-12.

14 Rashid A, Hamilton SR. Necrosis of gastrointestinal tract in uremic patients as a result of sodium polystyrene sulfonate (Kayexalate) in sorbitol: an underrecognized condition. Am f Surg Pathol 1997;21: 60-9.

15 Gennari FJ. Hypokalemia. N Engl f Med 1998;339:451-7.

16 Amlal H, Krane CM, Chen QK, et al. Early polyuria and urinary concentrating defect in potassium deprivation. $A m \mathcal{F}$ Physiol 2000;279:F655-63.

17 Alpern RJ, Emmett M, Seldin DW. Metabolic alkalosis. In: Seldin DW, Giebisch G, eds. The kidney: physiology and pathophysiology. 2nd Ed. New York: Raven Press, 1992: 2733-56.

18 Soleimani M, Bergman JA, Hosford MA, et al. Potassium depletion increases $\mathrm{Na}^{+}: \mathrm{CO}_{3}{ }^{=}: \mathrm{HCO}_{3}{ }^{-}$cotransport in rat renal cortex. F Clin Invest 1990;86:1076-83.

19 Amlal H, Habo K, Soleimani M. Potassium depletion upregulates the expression of the renal basolateral $\mathrm{Na}^{+}: \mathrm{HCO}_{3}{ }^{-}$ cotransporter (NBC-1). Am f Physiol 2000;279:F532-43.

20 Silver R, Soleimani M. H+-K+-ATPases: regulation and role in pathophysiologic states. Am f Physiol 1999;276:F799-811.

21 Amlal H, Wang Z, Soleimani M. Potassium depletion downregulates chloride-absorbing transporters in rat kidney. F Clin Invest 1998;101:1045-54. 
LETTERS

\section{The role of tricyclic antidepressants and tramadol in palliative care}

We read with interest the review article on alternative opioids to morphine in palliative care. ${ }^{1}$ The author has mentioned in detail various factors-biomedical, genetic, and psychological-which influence the effect of opioids. Though most of the aspects are wel covered, the role of depression has not been discussed, and this has a tremendous impac on the manifestation and management of pain. $^{2}$ Initial control of depression greatly facilitates pain management. Depression must be treated aggressively (for example with antidepressants and psychotherapy sessions) or pain management will remain elusive. It has also been shown that in patients who are taking opioid drugs, the bioavailability of opioids is increased with antidepressants; the tricyclic drugs are membrane stabilising, which may account for the early onset of action in patients with chronic pain.

The author mentioned the advantages of transdermal administration of fentanyl. She noted that it is highly acceptable to patients and the patches can be applied by patients or relatives themselves. We would like to stress that $25 \%$ to $50 \%$ of patients above the age of 65 suffer from major pain problems. Age related changes in skin integrity, subcutaneous fat, and water content can affect patien response to transdermal products. In fact, fentanyl patches have been associated with death in opioid-naive older adults in doses as low as $50 \mathrm{\mu g} /$ hour. $^{2}$ Also, serum fentanyl concentrations may increase by one third in patients with a body temperature of $40^{\circ} \mathrm{C}$ or more. It has been suggested that fentanyl should not be given to children younger than 12 years of age or to patients younger than 18 years of age who weigh less than $50 \mathrm{~kg}$. Additionally, fentanyl has a long duration of action (up to 72 hours) and therefore the side effects and adverse reactions are not easily reversed. In view of this, we believe that transdermal fentanyl should not be used liberally.

It was also stated by the author that tramadol is less potent than morphine and less effective for managing severe pain. However tramadol has been used extensively and evaluated over the last 20 years. It has proved as effective as the strong opioids in acute and chronic pain settings. In particular, tramadol administration results in little respiratory depression in comparison with equianalgesic doses of opioids, such as morphine or pethidine. Tramadol has a long record of efficacy and safety, and although it should be avoided or used with caution in epileptic patients, it is now the fourth most commonly prescribed analgesic worldwide. ${ }^{5}$ It is certainly useful in the treatment of chronic, non malignant, and malignant pain syndromes. Another considerable advantage of tramadol is its very low abuse potential. Consequently, it is not deemed a controlled (scheduled) drug.

In view of the above, we believe that tramadol has an important role as an alternative opioid to morphine in palliative care.
M Thulasimani

Department of Medicine Community Health Center, Mannadipet, Pondicherry 605501 , India Prakram@md4 vsnl.net.in

S Ramaswamy

Department of Clinical Pharmacology, Jawaharla Institute of Postgraduate Medical Education and Research, Pondicherry 605 006, India

\section{References}

1 Barnett M. Alternative opioids to morphine in palliative care: a review of current practice and evidence. Postgrad Med J

2001;77:371-8.

2 Gloth III FM. Geriatric pain. Factors that limit pain relief and increase complications. Geriatrics 2000;55:46-52

3 Hardy PA. Analgesics. In: Hardy PA, ed Chronic pain management: the essentials. London: Greenwich Medical Media, 1977 : Londo

4 Salemo E. Analgesics. In: Salemo E, ed. Pharmacology for health professionals. USA: Mosby, 1999: 124-53.

5 Bamigbade JA, Langford RM. Tramadol hydrochloride: an overview of current use. Hospital Medicine 1998;59:373-6.

\section{Author's reply}

First, I would like to address the apparen omission of a discussion of depression. I am entirely in agreement with the respondents' comments that depression has a major role in the manifestation and management of pain. However, in this review I was considering the differential factors influencing choice of opioid, not the assessment of pain per se. I pointed out at the outset that cancer pain was multifactorial, and that a thorough assessment was a prerequisite to successful management, but that the review was of strong opioids, and was beginning from the point at which these were considered appropriate treatment. I did include the effect of antidepressants in enhancing bioavailability of opioids in the text and in table 5, but in the context of drug interactions.

With regard to transdermal fentanyl: the authors appear to imply that in beginning with a discussion of its advantages, I was advocating liberal use of this drug and route. I went on to describe all its disadvantages, including its interpatient variability and long duration of action causing potential problems of overdose. I was attempting to provide a balanced overview of a drug that has proved very popular with patients and health professionals while cautioning on its overuse.

On the question of tramadol, I do not dispute that it is an effective analgesic and has a broad spectrum of clinical use. However, both from personal practice and my review of literature pertinent to palliative care, I concluded that its efficacy in the management of progressive severe cancer pain is less conclusively demonstrated, and thus questioned its role in palliative care. Respiratory depression is rarely an issue in palliative care, nor opioid abuse, although I accept that low abuse potential may facilitate adequate analgesic prescribing in chronic non-malignant pain.

\section{Watermelon poisoning}

Food borne diseases are a result of ingestion of foods contaminated by either infectious or toxic agents. These diseases are sometimes inaccurately referred to as "food poisoning", and they represent one of the most widespread and overwhelming public health probems of the modern world. Infants, children the elderly, and the immunocompromised are more commonly affected. ${ }^{1}$ Infection of six members of a family is described here.

The head of a family (61 years), his wife (59 years), their son (38 years), daughter in law (35 years), and two male grandchildren (14 and 11 years respectively) were admitted to hospital with gastroenteritis. About four hours earlier they had consumed pieces of freshly cut watermelon. During the past seven days they had consumed home cooked food and clear water from the domestic supply. The head of the family, who had received the lion's share of the fruit, was affected the most and was in a state of shock and acute renal failure. It took three days for his urinary output and renal parameters to improve. He was treated with intravenous fluids, ciprofloxacin, metronidazole, and other conservative measures for five days. Other members of the family had an uneventful stay in the hospital and were discharged on the second day after admission. The daughter in law, who had received the smallest share of the fruit, was affected the least and had just two or three loose stools in hospital. Haematology, urinalysis, and chest radiography of all the family members were normal. Blood biochemistry of the head of the family suggested uraemia and acidosis. Stool cultures of all members of the family grew an enteroinvasive variety of Escherichia coli, which was non-motile with non-lactose ferments.

We asked the fruit seller about the purchase of watermelon and this revealed the fact that watermelons can be made more colourful and sweet without cutting them open. Instead a long needle, into the core, can inject sweetener and colouring agents, three to four hours before sale. The nature of the injected agents was not revealed by the fruit seller for obvious reasons. Culture from the solution that had been injected (which had been prepared and stored in an earthenware bowl), also grew multiple colonies of the enteroinvasive variety of $E$ coli, which were biochemically lactose positive, non-motile, with non-lactose ferments

Diarrhoeal diseases have been commonly attributed to a pathogen contaminated water supply, but it is now recognised that food also plays an equally important part in $70 \%$ of such illnesses. Besides the usual foods, contamination has been reported in other foods such as raw fish, shellfish, bivalve molluscs (oysters, cockles, mussels), raw shrimp, pork, mixed d'oeuvre, crabs, prawns, rock lobster, cooked squid, turkey, street foods, eggs, egg salad, cold asparagus, aquatic plants, bottle feeds (for infants), ice creams, chocolates, candies, etc. The chief contaminants are bacteria ( $E$ coli, shigella, salmonella, Vibrio cholera 01, Campylobacter jejuni, brucella, Bacillus cereus, Staphylococcus aureus, Clostridium perfringens, and Clostridium botulinum), helminthes (Trichinella spiralis, Taenia saginata, Taenia solium, clonorchis, Fasciola opisthorchis Paragonimus spp), protozoa (Entamoeba hystolitica, Giardia lamblia, Cryptosporidium spp), and enteric viruses (rotavirus hepatitis A\&E virus) etc. ${ }^{1}$ 
Infections due to pathogenic strains of $E$ coli are probably the commonest cause of diarrhoea in developing countries. The contamination of food with micro-organisms is caused by:

- Use of contaminated equipment.

- Infected food handlers.

- Use of raw and contaminated ingredients.

- Cross contamination.

- Addition of toxic chemicals or use of foods containing natural toxicants like mushrooms etc. ${ }^{1}$

Gastroenteritis by Salmonella javiana contamination of watermelon has been described in 26 cases in the USA. ${ }^{2}$ Contamination of fruit by such a novel method as described here may prove to be a major public health hazard, and hence is noteworthy.

T K Pande, A H Khan, R Pipersania, S K Sethi, Y Rath

Department of Internal Medicine, J L N Hospital and Research Center, Bhilai 490 009, India; pandetk@mantraonline.com

\section{References}

1 World Health Organization. Food borne disease: a global health and economic problem. Food borne disease: a focus for health education. Geneva, Switzerland: WHO, 2000: 1-34.

2 Blostein J. An outbreak of Salmonella javiana associated with consumption of watermelon. Journal of Environmental Health 1993;56:29-31.

\section{BOOK REVIEWS}

The reviewers have been asked to rate these books in terms of four items: readability, how up to date they are, accuracy and reliability, and value for money, using simple four point scales. From their opinions we have derived an overall "star" rating: * $=$ poor, ${ }^{* *}=$ reasonable, ${ }^{* * *}=$ good, ${ }^{* * * *}=$ excellent.

\section{A Practical Guide for Medical Teachers.}

Edited by John A Dent and Ronald $M$ Harden. (Pp 453; £34.95.) Churchill Livingstone 2001. ISBN 0-443-06273-0. *** *

This book tells you all you wanted to know and a good deal more that you now need to know about the revolution that has been taking place in undergraduate medical education in the past decade or more. The editors' stated intention is to provide jargon-free understanding of contemporary educational principles and provide practical advice on dealing with all aspects of teaching. They succeed brilliantly well.

The book is divided into seven sections (totalling 39 chapters in all) starting with aspects of the curriculum, learning situations such as lectures, small groups, wards, primary care etc, educational strategies (independent, problem based, integrated and multiprofessional), aids such as computers, audiovideo, and guides, themes (basic sciences, communication skills, ethics, informatics etc), means of assessment, and aspects of students and staff such as selection, support staff development, and course monitoring.
There is evidence of tight editing in terms of chapter style and layout and excellent cross referencing between the chapters. The book is enlivened by quotations in wide easy-on-theeye margins. These also host simple line drawings of such important practical aspects of teaching as the ideal distribution of teacher and taught round a bed. The advice to ensure students do not go off on electives to countries with military or civil unrest is not profound but well timed. Each chapter provides suggestions for further reading.

The principles and examples are, as the editors suggest, relevant to postgraduate medical education and other healthcare professionals. But for those involved in the teaching of medical students, this book from Dundee, a teaching centre of excellence, is warmly recommended for those who want to improve their understanding and performance and hence enjoyment of this Hippocratic responsibility.

B I Hoffbrand Fellowship of Postgraduate Medicine, London, UK

\section{Making Sense of Statistics and Healthcare.}

By Anna Hart. (Pp 162; £19.95.) Radcliffe Medical Press, 2001. ISBN 1-85775-472Medical

This book describes the essentials of statistics and data presentation. The first chapters deal with the different types of study designs, the various forms of data and the characteristics of pictorial forms of presenting data. Subsequent chapters deal with distributions, means and medians, and measures of variability. The author describes confidence intervals, p values, statistical power, and type I and II errors. The final sections are on confounders, interactions, measures of correlation, hierarchy of evidence, causality, and how to assess papers. The author describes the terms used in evidence based medicine such as absolute risks and the number needed to treat. A glossary of terms used is contained in the appendices.

The book will be of invaluable use for those just starting a research career or in its early stages. It is comprehensive and clearly written with many illustrative examples. The text covers well all the areas that are of interest to those commencing their own research projects. The pitfalls of different tests are well described and the book is competitively priced.

A R Hart

Consultant Gastroenterologist West Norwich Hospital, UK

\section{Fast Facts-Sexually Transmitted Infections.}

By Ann Edwards, Jackie Sherrard, and Jonathan Zenilman. (Pp 104; $£ 12.00$.) Health Press, 2001. ISBN 1-899541-04-7.****

In a month when all GPs in my area have been notified of an epidemic of syphilis in London, the publication of this book could not be more timely.

There has long been a need for a concise, affordable, accessible, and accurate textbook on sexually transmitted infections (STIs) for non-specialists in genitourinary medicine and this volume fills the gap perfectly. The clear, sharp, and relevant photographs and illustrations are an added bonus and the tables such as those of causes of vaginal discharge and genital ulceration are exemplary in their clarity and comprehensiveness. Relevant aspects of dermatology and even psychiatry are briefly considered where appropriate.

Three opening chapters on aetiology of STIs, taking a sexual history and counselling approaches, are followed by chapters dealing with STIs causes by bacterial, viral, and other organisms in turn. For once a correct balance (in terms of their respective incidence in the UK) is shown between coverage of HIV and non-HIV STIs. Other chapters are problem oriented and consider such commonly presenting conditions as urethritis and pelvic inflammatory disease. Comprehensive treatment guidelines for both the UK and USA conclude the book.

As STIs continue to rise in the UK, this text will prove an indispensable ready reference guide for paediatricians, physicians, GPs, gynaecologists, dermatologists, and surgeons. I will add my copy to the other three essential reference books on my surgery desk.

T G Stammers General Practitioner, Merton Park, London, UK

\section{MCQs in Paediatrics for MRCPCH Part I.}

By Nagi G Barakat. (Pp 212; £14.95.) Royal Society of Medicine Press, 2001. ISBN 1-85315-491-1. **

This paperback book in fact has 463 questions including a 60 question examination. The format is of MCQs as used in the part I of the MRCPCH examination and the question material does cover the full syllabus for this. The answers provide some explanations for the questions but not all. The evidence for the answers isn't related to specific text. However, there are a number of recommended texts and references listed, but at least one of these texts, that for neonatology, does not show the most recent edition edited by Roberton and Rennie.

As with all MCQ questions, some of the answers are arguable but there are certain answers given that are clearly completely wrong, which will further mislead the examination candidate.

Although the material covers the syllabus, the questions that have been prepared do not reflect the quality of the questions expected in the membership examination.

Given the thirst for examination questions among membership candidates, however, it is likely that some candidates will find the book useful in their preparation.

A C Elias-Jones Consultant Paediatrician Leicester General Hospital, and Examination Committee,

Royal College of Paediatrics and Child Health, UK

\section{Imaging Picture Tests for the MRCPCH.}

By A P Winrow. (Pp 205; £ 19.95.) Churchill Livingstone, 2000. ISBN 0-443-06445-

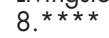

This is a useful paediatric teaching book for candidates studying for the MRCPCH final examination. It may also be useful for 
candidates for the Diploma for Child Health. It contains a selection of 100 varied paediatric and neonatal cases of the types commonly seen in the examination. There is an easily accessible question and answer format on the following page with useful comments relevant to the condition being demonstrated There are also useful examination tips applied to each case

The author admits that some of the images were obtained from copy film and as a result some of the images are perhaps not as clear a they might have been. However, the examination candidate with the help of the answers and comments should be able to follow the demonstrated abnormalities.

The examination candidates will find this a useful additional text to assist their learning. It could also provide useful CPD for self assessment by already qualified paediatricians in addition.

A C Elias-Jones

Consultant Paediatrician Leicester General Hospital,

and Examination Committee

Royal College of Paediatrics and Child Health, UK

\section{DIARY}

\section{Fellowship of Postgraduate \\ Medicine \& CHIME, Royal Free \& \\ University College Medical \\ School}

\section{Clinical Governance, Thursday, 21 \\ February 2002}

A one day conference for doctors wishing to become more involved in clinical governance at the Commonwealth Conference Centre, Kensington High Street, London.

\section{PACES (MRCP) Part II}

Two two day courses designed as final preparation for the PACES examination to be held at the Whittington Hospital, London.

Communication Skills and Ethics: 7 and 8 February 2002. Cost: $£ 500$.

Clinical: 12 and 13 February 2002. Cost: $£ 500$. For details contact Kate Stephens, CHIME, 4th Floor Holborn Union Building, Archway Campus, Highgate Hill, London N19 3UA (tel: 020-7288-3134,email: k.stephens@chime. ucl.ac.uk)

\section{Thackray Museum}

25 April 2002-History of herbals, a talk by Bruce Madge of the British Library.

11 May 2002-Use and abuse: a history of opium, University of Leeds Medical History Day School.

For further information contact the Thackray Museum, Beckett Street, Leeds LS9 7LN (tel: 0113244 4343, fax: 01132470219 , email: info@thackraymuseum.org, web site: thack raymuseum.org).

\section{CORRECTION}

\section{Hypokalaemia and hyperkalaemia}

An error occurred in the above paper, published in the December issue of the journal (2001;77:759-64). The name of the first author was spelt incorrectly; the correct spelling is A Rastegar. 